\title{
Ideação suicida na adolescência: prevalência e fatores associados
}

\author{
Suicidal ideation in adolescence: prevalence and associated factors \\ Luciano Dias de Mattos Souza', Liliane Ores², Gabriela Teixeira de Oliveira², Ana Laura Sica Cruzeiro², \\ Ricardo Azevedo Silva ${ }^{3}$, Ricardo Tavares Pinheiro ${ }^{4}$, Bernardo Lessa Horta ${ }^{5}$
}

\section{RESUMO}

\section{Palavras-chave}

Ideação suicida, suicídio, adolescência, saúde mental.

\section{Keywords}

Suicidal ideation, suicide, adolescence, mental health.
Objetivo: Avaliar a prevalência de ideação suicida, assim como de seus fatores associados, em adolescentes entre 15 e 18 anos da cidade de Pelotas (RS). Métodos: 960 adolescentes responderam a um questionário autoaplicado e sigiloso em estudo transversal de base populacional. A ideação suicida foi aferida por meio do item 17 do SRQ-20, que avalia transtornos psiquiátricos menores. Para a análise estatística, utilizou-se a regressão logística. Resultados: A prevalência de ideação suicida foi de 7,7\%. A baixa escolaridade da mãe, a baixa escolaridade do adolescente, o sedentarismo, o uso de álcool e de outras substâncias e o comportamento agressivo mantiveram associação estatisticamente significativa com ideação suicida. Conclusão: Programas preventivos devem preferencialmente atingir adolescentes de baixa escolaridade que apresentem comportamento agressivo e relatem uso de substâncias.

\section{ABSTRACT}

Objective: To assess the prevalence of suicidal ideation and associated factors in adolescents between 15 and 18 years of Pelotas (RS). Methods: 960 adolescents answered a self-areported and confidential questionnaire in a population-based cross-sectional study. Suicidal ideation was measured by item 17 of the SRQ-20 that assesses minor psychiatric disorders. Statistical analysis used the logistic regression. Results: The prevalence of suicidal ideation was 7.7\%. Low mother's education, low schooling, physical inactivity, alcohol and other substances and aggressive behavior remained significantly associated with suicidal ideation after multivariate analysis. Conclusion: Prevention programs should preferably reach adolescents with low education who have aggressive behavior and report use of substances.

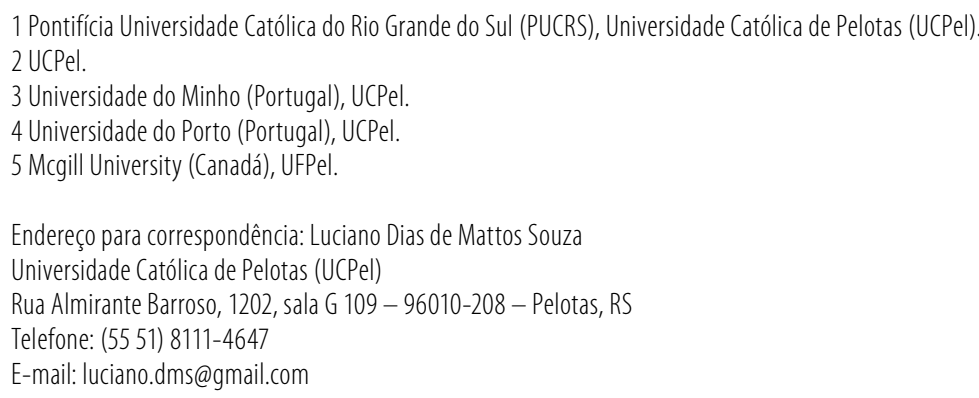




\section{INTRODUÇÃO}

Os elevados índices de suicídio, tentativas de suicídio e ideação suicida caracterizam tal tema como um importante problema de saúde pública'. A adolescência, por sua vez, é o grupo etário que mais mobiliza preocupações com comportamentos de risco à saúde².

O suicídio é a terceira principal causa de morte na adolescência ${ }^{3}$. A ideação suicida consiste em um preditor de tentativas de suicídio ${ }^{4}$ e, nesse sentido, pode ser considerada como primeiro passo para que tal atitude seja finalizada ${ }^{5}$.

Na Malásia, um estudo transversal com 4.500 estudantes adolescentes apontou que 7\% dos participantes tinham considerado a tentativa de suicídio e 4,6\% já haviam tentado suicídio nos últimos 12 meses. Adolescentes do sexo feminino se apresentaram mais propensas aos comportamentos suicidas, bem como aqueles que relataram maior número de dias de ausência na escola, envolvimento em luta física e ter dirigido veículo depois de ingerir bebida alcoólica no último ano ${ }^{6}$.

No âmbito nacional, em estudo transversal realizado em município da região metropolitana de Porto Alegre, Rio Grande do Sul, com escolares entre 12 e 18 anos, foi encontrada prevalência de $6,3 \%$ de planejamento suicida. As meninas foram mais propensas a planejarem o suicídio do que os meninos. Da mesma forma, os jovens que relataram problemas na relação com os pais, pequeno número de amigos, relações agressivas com amigos e sentimentos de tristeza e solidão também apresentaram maior risco de planejamento suicida ${ }^{7}$. No mesmo sentido, Silva et al. ${ }^{8}$, em estudo de caso controle, observaram que a ideação suicida esteve associada com sintomas depressivos, não prática religiosa e vizinhança não solidária ${ }^{8}$.

Mesmo nos casos em que o suicídio não é completado, a ideação suicida parece relacionar-se com consequências negativas, uma vez que está associada a um maior risco de transtornos psiquiátricos, problemas comportamentais, baixa autoestima, pobres habilidades em enfrentar problemas e relacionamentos interpessoais na vida adulta?

No Brasil, os acidentes e as violências representam um relevante problema de saúde pública que tem causado intenso impacto na morbidade e na mortalidade da população. Por isso, existem esforços e políticas públicas destinadas ao tema. As diretrizes da Política Nacional de Redução da Morbimortalidade por Acidentes e Violências buscam, por meio de suas ações, a promoção de comportamentos imunógenos e de ambientes seguros; o acompanhamento dos índices de acidentes e de violências; o aperfeiçoamento do atendimento pré-hospitalar, assim como a assistência às vítimas de tais desfechos. Da mesma forma, esses esforços ainda buscam capacitar recursos humanos sobre tais demandas e auxiliar na elaboração de investigações sobre esses temas ${ }^{10}$.

No mesmo sentido, em outubro de 2006, foi publicado o Manual de Prevenção ao Suicídio, dirigido aos profissio- nais das equipes de saúde mental ${ }^{11}$, com o interesse de prevenção de comportamentos suicidas. Esse documento tem como objetivo transmitir informações básicas para orientar na detecção precoce de condições mentais relacionadas ao comportamento suicida, como também informa sobre o manejo inicial de pessoas que possuem risco de suicídio e medidas de prevenção. Contudo, como previsto nas iniciativas governamentais acima expostas, mais estudos são necessários, com o objetivo de alcançar informações e estratégias que colaborem para a redução da mortalidade e morbidade da população, reduzindo as taxas de violência e acidentes no Brasil. Estratégias de prevenção congruentes ao contexto nacional são necessárias. Assim, o presente estudo avaliou a prevalência de ideação suicida em jovens de 15 a 18 anos da cidade de Pelotas (RS) no ano de 2002, bem como seus fatores associados.

\section{MÉTODOS}

No ano de 2002, realizou-se estudo transversal em uma amostra representativa de jovens com idades entre 15 e 18 anos, residentes na zona urbana da cidade de Pelotas (RS). Essa investigação foi aprovada pelo Comitê de Ética em Pesquisa do Hospital Santa Casa de Misericórdia de Pelotas.

Foram selecionados aleatoriamente 90 dos 448 setores censitários da zona urbana de Pelotas. Em cada um desses setores, mediante sorteio, foram indicados um quarteirão e uma esquina como ponto inicial. A partir desse ponto, 86 residências foram sistematicamente visitadas em cada setor. No total, 7.740 domicílios foram visitados pela equipe de pesquisa.

O tamanho da amostra foi calculado para um estudo amplo sobre comportamentos de saúde na adolescência. Em função dos seus múltiplos objetivos, o tamanho da amostra calculado por meio do programa Epilnfo 6.4 foi de 800 adolescentes.

Após a obtenção do consentimento por escrito de um dos pais ou responsável pelo adolescente, o jovem respondia a um questionário autoaplicado e sigiloso com questões estruturadas sobre sexo, idade, nível socioeconômico, escolaridade dos pais, condições de moradia, nível educacional, trabalho remunerado, atividade física, uso de substâncias (drogas ilícitas, tabaco e álcool), comportamento agressivo e ao Self Reporting Questionnaire (SRQ-20) ${ }^{12}$ - para aferição da presença de transtornos psiquiátricos menores. O referido instrumento foi elaborado pela Organização Mundial de Saúde para a detecção de morbidade psiquiátrica na população geral e consiste de 20 questões com duas possibilidades de resposta (sim/não) cada. No que diz respeito à ideação suicida, o referido desfecho foi avaliado por meio do item 17 da escala SRQ-20. Nesse item, consta a pergunta: "Tens tido a ideia de acabar com a vida?". Foi caracterizada ideação sui- 
cida nos adolescentes que assinalaram "Sim" como resposta a esse item. Para avaliar o nível socioeconômico, utilizou-se a classificação da Associação Brasileira dos Institutos de Pesquisa de Mercado (Abipeme) ${ }^{13}$. Por fim, depositava-se o questionário em uma urna lacrada.

Foram feitas duas digitações no programa Epi-Info $6.4 \mathrm{e}$ estas foram comparadas para que as inconsistências fossem resolvidas. O programa Stata foi utilizado na análise estatística.

Para a análise estatística, utilizou-se a regressão logística, que foi utilizada também para análise bivariada e multivariada. Nesta última, seguiu-se o modelo hierárquico ilustrado pela figura 1. As variáveis independentes que na análise bivariada apresentaram um valor $\mathrm{p} \leq 0,20$ foram incluídas no modelo proposto. Na execução da análise multivariada, inicialmente se verificou a associação do desfecho de ideação suicida com as variáveis do primeiro nível. Permaneceram no modelo aquelas variáveis que apresentaram valor $p \leq 0,20$ (sexo, idade, escolaridade da mãe). Posteriormente, foram inseridas as variáveis do segundo nível (escolaridade do adolescente e sedentarismo), que permaneceram no modelo hierárquico por apresentarem valor $p \leq 0,20$. Por fim, foram inseridas as variáveis do terceiro nível, mantendo-se no modelo de análise apenas aquelas que apresentaram valor $p \leq 0,20$.

\begin{tabular}{|c|}
\hline $\begin{array}{l}\text { 10 nível } \\
\text { Sexo } \\
\text { Idade } \\
\text { Escolaridade da mãe }\end{array}$ \\
\hline $\begin{array}{l}2^{20} \text { nível } \\
\text { Escolaridade do adolescente } \\
\text { Sedentarismo }\end{array}$ \\
\hline $\begin{array}{l}\text { 3o nível } \\
\text { Usou drogas no último mês } \\
\text { Tomou porre no último mês } \\
\text { Fumou no último mês } \\
\text { Carregou arma nos últimos } 12 \text { meses } \\
\text { Participou de briga com agressão nos últimos } 12 \text { meses }\end{array}$ \\
\hline
\end{tabular}

Figura 1. Diagramação do modelo hierarquizado final de análise multivariada dos dados.

\section{RESULTADOS}

Um total de 1.039 jovens foi identificado; as perdas ou recusas representaram 7,6\% da amostra e ocorreram em virtude de o adolescente não estar em casa após pelo menos três visitas do aplicador ou por não haver a concordância dos pais ou responsáveis com a realização da entrevista. A presente investigação avaliou uma amostra representativa de 953 adolescentes residentes na zona urbana de Pelotas (RS) e com idades entre 15 e 18 anos, pois em sete questionários a questão referente à ideação suicida não foi respondida.
A tabela 1 apresenta a caracterização dos jovens entrevistados. Do total de entrevistados, 51,8\% eram do sexo feminino, 38,0\% foram classificados como sendo de classe C e 58,0\% relataram escolaridade de nove anos de estudo ou mais. A maioria dos participantes relatou morar com pai e/ou com mãe, e a escolaridade de até oito anos de estudo foi observada em maior proporção. Cerca de um quinto dos adolescentes assinalou que trabalha de forma remunerada, enquanto $64,3 \%$ afirmaram praticar sua religião. No que tange ao consumo de substâncias, o uso de álcool no último mês foi observado em $42,9 \%$ da amostra e 6,4\% desta afirmaram ter tomado um porre no mesmo período. O consumo de tabaco e drogas foi relatado por $16,3 \%$ e 8,4\%, respectivamente. A maioria dos jovens não relatou participação em briga com agressão física no último ano e 9,5\% dos entrevistados afirmaram ter carregado arma nesse período. Com relação ao desfecho em estudo, foi observada prevalência de 7,7\% de ideação suicida.

Tabela 1. Descrição dos adolescentes de 15 a 18 anos de Pelotas (RS), no ano de 2002

\begin{tabular}{|c|c|c|}
\hline & $\mathrm{N}$ & $\%$ \\
\hline \multicolumn{3}{|l|}{ Sexo } \\
\hline Masculino & 459 & 48,2 \\
\hline Feminino & 494 & 51,8 \\
\hline \multicolumn{3}{|c|}{ Nível socioeconômico } \\
\hline A ou $B$ & 343 & 36,0 \\
\hline C & 362 & 38,0 \\
\hline DouE & 248 & 26,0 \\
\hline \multicolumn{3}{|l|}{ Idade } \\
\hline 15 & 227 & 23,8 \\
\hline 16 & 267 & 28,0 \\
\hline 17 & 219 & 23,0 \\
\hline 18 & 240 & 25,2 \\
\hline \multicolumn{3}{|c|}{ Escolaridade materna } \\
\hline Até 4 anos & 199 & 20,9 \\
\hline De 5 a 8 anos & 411 & 43,1 \\
\hline 9 ou mais anos & 343 & 36,0 \\
\hline \multicolumn{3}{|c|}{ Escolaridade paterna } \\
\hline Até 4 anos & 211 & 22,1 \\
\hline De 5 a 8 anos & 402 & 42,2 \\
\hline 9 ou mais anos & 340 & 35,7 \\
\hline \multicolumn{3}{|c|}{ Escolaridade do adolescente } \\
\hline Até 4 anos & 51 & 5,4 \\
\hline De 5 a 8 anos & 349 & 36,6 \\
\hline 9 ou mais anos & 553 & 58,0 \\
\hline \multicolumn{3}{|l|}{ Pai mora em casa } \\
\hline Sim & 610 & 64,0 \\
\hline Não & 343 & 36,0 \\
\hline \multicolumn{3}{|l|}{ Mãe mora em casa } \\
\hline Sim & 800 & 83,9 \\
\hline Não & 153 & 16,1 \\
\hline
\end{tabular}


Tabela 1. Descrição dos adolescentes de 15 a 18 anos de Pelotas (RS), no ano de 2002 (continuação)

\begin{tabular}{|c|c|c|}
\hline & $\mathrm{N}$ & $\%$ \\
\hline \multicolumn{3}{|l|}{ Trabalho remunerado } \\
\hline Sim & 199 & 20,9 \\
\hline Não & 754 & 79,1 \\
\hline \multicolumn{3}{|l|}{ Pratica religião } \\
\hline Sim & 613 & 64,3 \\
\hline Não & 340 & 35,7 \\
\hline \multicolumn{3}{|c|}{ Bebeu álcool no último mês* } \\
\hline Sim & 406 & 42,9 \\
\hline Não & 540 & 57,1 \\
\hline \multicolumn{3}{|c|}{ Tomou porre no último mês* } \\
\hline Sim & 60 & 6,4 \\
\hline Não & 873 & 93,6 \\
\hline \multicolumn{3}{|c|}{ Fumou tabaco no último mês } \\
\hline Sim & 155 & 16,3 \\
\hline Não & 798 & 83,7 \\
\hline \multicolumn{3}{|c|}{ Usou drogas ilícitas no último mês } \\
\hline Sim & 80 & 8,4 \\
\hline Não & 873 & 91,6 \\
\hline \multicolumn{3}{|l|}{ Sedentarismo } \\
\hline Sim & 371 & 38,9 \\
\hline Não & 582 & 61,1 \\
\hline \multicolumn{3}{|c|}{ Carregou arma nos últimos 12 meses* } \\
\hline Sim & 89 & 9,5 \\
\hline Não & 852 & 90,5 \\
\hline \multicolumn{3}{|c|}{ Participou de briga com agressão nos últimos 12 meses* } \\
\hline Nenhuma & 731 & 77,4 \\
\hline Uma briga & 96 & 10,2 \\
\hline Duas ou mais brigas & 118 & 12,5 \\
\hline \multicolumn{3}{|l|}{ Ideação suicida } \\
\hline Sim & 73 & 7,7 \\
\hline Não & 880 & 92,3 \\
\hline
\end{tabular}

* Foram utilizados os percentuais válidos devido à existência de não resposta na população.

Conforme a tabela 2, inicialmente, a ideação suicida esteve associada ao sexo feminino (OR 1,64 IC 95\% 1,00 a 2,69), baixa escolaridade do pai e da mãe dos jovens, bem como com a escolaridade do próprio adolescente. Nessas variáveis, os grupos que assinalaram até quatro anos de estudo apresentaram proporção significativamente maior de ideação suicida do que os grupos com escolaridade de nove anos ou mais de estudo $(p<0,050)$. No que diz respeito aos comportamentos relacionados à saúde dos entrevistados, o desfecho em estudo mostrou-se associado ao sedentarismo (OR 2,01 IC 95\% 1,24 a 3,25), ter consumido álcool (OR 1,84 IC 95\% 1,13 a 3,00), drogas (OR 2,93 IC 95\% 1,55 a 5,52) e ter tomado porre no último mês (OR 3,96 IC 95\% 2,03 a 7,74), assim como ter carregado revólver, faca ou canivete (OR 2,60 IC 95\% 1,39 a 4,89) e ter se envolvido em duas ou mais brigas com agressão nos últimos 12 meses (OR 2,60 IC 95\% 1,37 a 4,94).
Após o ajuste para os possíveis fatores de confusão investigados, o desfecho se manteve significativamente associado a baixa escolaridade materna, baixa escolaridade do adolescente, sedentarismo, uso de drogas no útlimo mês bem como ter tomado "porre" no mesmo período, carregado revólver, faca ou canivete e ter se envolvido em duas ou mais brigas com agressão nos últimos 12 meses $(p<0,050)$. O grupo de jovens que assinalou escolaridade materna de até quatro anos de estudo apresentou proporção de ideação suicida 93\% maior do que aqueles que relataram a escolaridade materna de nove ou mais anos de estudo. No mesmo sentido, os jovens com baixa escolaridade apresentaram proporção três vezes maior de ideação suicida em relação ao grupo de adolescentes com nove anos ou mais de estudo. Os participantes considerados sedentários apresentaram risco 71\% maior para ideação suicida do que os demais jovens. Jovens que relataram ter tomado porre no último mês apresentaram risco cinco vezes maior de ideação suicida do que aqueles que não relataram tal comportamento (OR 5,00 IC 95\% 2,28 a 10,95). Da mesma forma, o uso de drogas apresentou-se como variável significativamente associada ao desfecho estudado (OR 2,40 IC 95\% 1,12 a 5,14). Ter carregado arma (OR 3,30 IC 95\% 1,43 a 7,65) e ter se envolvido em duas ou mais brigas com agressão (OR 2,52 IC 95\% 1,22 a 5,21 ) apresentou risco acrescido para ideação suicida. Assim, os adolescentes que relataram comportamentos agressivos apresentaram ideação suicida em maior proporção quando comparados com os participantes que não apresentaram tais comportamentos (Tabela 2).

\section{DISCUSSÃO}

A presente investigação constitui parte importante no processo de construção do conhecimento sobre a suicidabilidade na adolescência. Do total de 960 adolescentes entrevistados, encontrou-se uma prevalência de ideação suicida de $7,7 \%$. No que tange à ideação suicida ao longo da vida, as prevalências encontradas na literatura científica são mais elevadas. Em pesquisa realizada com 526 adolescentes entre 15 e 19 anos de escolas públicas e privadas de Porto Alegre, 35,7\% apresentaram ideação na Escala de Ideação Suicida de Beck (BSI) ${ }^{14}$. Em estudo populacional realizado em Campinas (SP) com 515 indivíduos $\geq 14$ anos de idade, a prevalência de ideação suicida ao longo da vida foi de $17,1 \%$ e de 5,3\% ao longo dos últimos 12 meses. Na referida pesquisa, a ideação suicida foi avaliada utilizando-se uma entrevista padronizada, da Organização Mundial de Saúde (OMS), sobre comportamento suicida ${ }^{15}$. Estudo de base populacional, com uma população de 10.414 adolescentes entre 14 e 16 anos, realizado em Pernambuco, resultou que foi referida a ideação suicida/tentativa de suicídio por 34,3\% dos adolescentes entrevistados $^{16}$. 
Tabela 2. Modelo hierárquico final para ideação suicida por regressão logística (razão de odds ratio e 95\% de intervalo de confiança), Pelotas (RS), 2002

\begin{tabular}{|c|c|c|c|c|}
\hline Variável & OR bruto (IC) & p-valor & OR ajustado (IC) & p-valor \\
\hline \multicolumn{5}{|l|}{ Primeiro nível } \\
\hline \multicolumn{5}{|l|}{ Sexo } \\
\hline Masculino & Referência & & Referência & \\
\hline Feminino & $1,64(1,00$ a 2,69$)$ & 0,049 & $1,61(0,98$ a 2,64) & 0,060 \\
\hline \multicolumn{5}{|l|}{ Nível socioeconômico** } \\
\hline A ou B & Referência & & & \\
\hline C & $1,28(0,68$ a 2,4$)$ & 0,442 & & \\
\hline D ou E & $1,37(0,78$ a 2,41$)$ & 0,281 & & \\
\hline \multicolumn{5}{|l|}{ Idade } \\
\hline 15 & Referência & & Referência & \\
\hline 16 & $1,06(0,48$ a 2,34$)$ & 0,884 & $1,08(0,49$ a 2,40$)$ & 0,844 \\
\hline 17 & $1,88(0,94$ a 3,75$)$ & 0,072 & $1,84(0,92$ a 3,68$)$ & 0,086 \\
\hline 18 & $1,85(0,90$ a 3,79$)$ & 0,093 & $1,88(0,92$ a 3,87$)$ & 0,086 \\
\hline \multicolumn{5}{|l|}{ Escolaridade do $\mathrm{pai}^{* * *}$} \\
\hline Até 4 anos & $1,81(1,01$ a 3,24$)$ & 0,046 & $1,40(0,70$ a 2,80$)$ & 0,337 \\
\hline De 5 a 8 anos & $1,67(0,85$ a 3,28$)$ & 0,139 & $1,26(0,56$ a 2,82$)$ & 0,579 \\
\hline 9 anos ou mais & Referência & & Referência & \\
\hline \multicolumn{5}{|l|}{ Escolaridade da mãe } \\
\hline Até 4 anos & $2,01(1,12$ a 3,62$)$ & 0,020 & $1,93(1,07$ a 3,50$)$ & 0,030 \\
\hline De 5 a 8 anos & $1,79(0,89$ a 3,59$)$ & 0,101 & $1,70(0,84$ a 3,42$)$ & 0,139 \\
\hline 9 anos ou mais & Referência & & Referência & \\
\hline \multicolumn{5}{|l|}{ Segundo nivel } \\
\hline \multicolumn{5}{|c|}{ Escolaridade do adolescente } \\
\hline Até 4 anos & $3,08(1,39$ a 6,81$)$ & 0,006 & $2,95(1,22$ a 7,12$)$ & 0,016 \\
\hline De 5 a 8 anos & $1,25(0,75$ a 2,09$)$ & 0,390 & $1,14(0,65$ a 2,01$)$ & 0,656 \\
\hline 9 anos ou mais & Referência & & Referência & \\
\hline \multicolumn{5}{|l|}{ Mora com a mãe $e^{* *}$} \\
\hline $\operatorname{Sim}$ & Referência & & & \\
\hline Não & $1,39(0,77$ a 2,52$)$ & 0,279 & & \\
\hline \multicolumn{5}{|l|}{ Mora com o pai** } \\
\hline Sim & Referência & & & \\
\hline Não & $1,05(0,64$ a 1,72) & 0,854 & & \\
\hline \multicolumn{5}{|l|}{ Trabalho remunerado** } \\
\hline $\operatorname{Sim}$ & Referência & & & \\
\hline Não & $0,94(0,53$ a 1,67$)$ & 0,821 & & \\
\hline \multicolumn{5}{|l|}{ Pratica religião** } \\
\hline Sim & Referência & & & \\
\hline Não & $1,00(0,61$ a 1,65$)$ & 0,991 & & \\
\hline \multicolumn{5}{|l|}{ Sedentarismo } \\
\hline Sim & $2,01(1,24$ a 3,25$)$ & 0,004 & $1,71(1,01$ a 2,92$)$ & 0,047 \\
\hline Não & Referência & & Referência & \\
\hline \multicolumn{5}{|l|}{ Terceiro nivel } \\
\hline \multicolumn{5}{|c|}{ Uso de drogas no último mês } \\
\hline Sim & $2,93(1,55$ a 5,52$)$ & 0,001 & $2,40(1,12$ a 5,14$)$ & 0,024 \\
\hline Não & Referência & & Referência & \\
\hline \multicolumn{5}{|c|}{ Uso de bebida alcoólica no último mês*** } \\
\hline Sim & $1,84(1,13$ a 3,00$)$ & 0,014 & $1,31(0,72$ a 2,40$)$ & 0,381 \\
\hline Não & Referência & & Referência & \\
\hline Tomou porre no último & & & & \\
\hline Sim & $3,96(2,03$ a 7,74$)$ & 0,000 & $5,00(2,28$ a 10,95$)$ & 0,000 \\
\hline Não & Referência & & Referência & \\
\hline Fumou no último mês & & & & \\
\hline Sim & $1,63(0,92$ a 2,89$)$ & 0,093 & $1,85(0,86$ a 3,96$)$ & 0,114 \\
\hline Não & Referência & & Referência & \\
\hline Carregou arma nos últin & & & & \\
\hline Sim & $2,60(1,39$ a 4,89$)$ & 0,003 & $3,30(1,43$ a 7,65$)$ & 0,005 \\
\hline Não & Referência & & Referência & \\
\hline Participou de briga com & & & & \\
\hline Nenhuma & Referência & & Referência & \\
\hline Uma briga & $1,89(0,98$ a 3,62$)$ & 0,056 & $1,04(0,45$ a 2,41$)$ & 0,919 \\
\hline Duas ou mais brigas & $2,60(1,37$ a 4,94$)$ & 0,004 & $2,52(1,22$ a 5,21$)$ & 0,013 \\
\hline
\end{tabular}

* Variável analisada de acordo com o percentual válido.

** Variável não controlada na análise ajustada.

*** Variável retirada do modelo hierárquico na análise ajustada. 
Dados de prevalência do desfecho estudado também oscilam quando este se refere ao último ano. Em um estudo transversal na Zâmbia com 1.970 estudantes, os quais participaram da Global School-based Student Health Survey (GSHS), 31,3\% deles relataram ideação suicida nos últimos 12 meses prévios à entrevista. A GSHS foi desenvolvida pela OMS, em colaboração com as Nações Unidas e com a assistência técnica dos Centers for Disease Control and Prevention (CDC), em Atlanta, EUA ${ }^{17}$. Dos 1.197 adolescentes entrevistados na Guiana, 18,4\% relataram ter seriamente considerado suicídio no último ano, avaliados por meio da GSHS ${ }^{18}$. No que se refere à ideação suicida nas últimas duas semanas, Souza et al. ${ }^{19}$ avaliaram jovens de 11 a 15 anos da cidade de Pelotas (RS), mediante um item da escala CDI (Child Depression Inventory), identificando prevalência de 14,1\%.

Além da diferença temporal para a ideação suicida, a variação entre os dados de prevalência entre os estudos acima descritos pode ser explicada pelas diferenças metodológicas na aferição da ideação suicida, na seleção da amostra, além de fatores socioculturais. Embora existam formas mais minuciosas de avaliar a ideação suicida em jovens, o presente estudo é caracterizado pela representatividade da amostra, ao contrário da maioria das pesquisas com adolescentes, desenvolvidas nas escolas, que podem ocasionar um viés de seleção. Ainda é relevante lembrar que o SRQ-20 foi validado para o Brasil e consiste em uma valiosa ferramenta para estudos de epidemiologia psiquiátrica, indicado para estudos de base populacional.

A baixa escolaridade da mãe, a baixa escolaridade do adolescente, o sedentarismo, o uso de álcool e de outras substâncias e o comportamento agressivo mantiveram associação estatisticamente significativa com ideação suicida. Cabe salientar a tendência estatística de associação significativa da ideação suicida com o sexo feminino, dado que corrobora achados de outros estudos $s^{6,7}$.

Os dados apoiam que adolescentes que possuem mãe com baixa escolaridade são mais propensos a ideias suicidas. Da mesma forma, adolescentes com baixa escolaridade possuem índices mais elevados de ideação suicida. Conforme An et al..$^{20}$, em estudo de base populacional com uma amostra de 2.965 adolescentes entre 15 e 18 anos e seus pais, pais e mães de adolescentes com ideação suicida não possuem alto grau de escolaridade. Tal fato parece ser congruente com o contexto encontrado na população geral. Em estudo com 14.104 homens e 16.562 mulheres com idade superior a 14 anos, a relação entre maior nível de escolaridade e menor prevalência de ideação suicida foi também observada²1.

No que se refere à saúde dos adolescentes, observou-se significância na correlação entre sedentarismo e ideação suicida. De acordo com Taliaferro et al., a participação dos adolescentes em atividades esportivas é um fator importante contra a depressão e a ideação suicida22. Babiss e Gangwisch afirmam que esportes possibilitam um aumento da autoesti- ma e do apoio social, assim como também elevam os níveis de endorfina e melhoram a imagem corporal ${ }^{23}$.

Quanto ao consumo de substâncias, Juan et al., ao realizarem estudo na China com estudantes de 12 a 19 anos, destacaram o uso de álcool e drogas como fator relacionado à ideação suicida, principalmente no início da adolescên$\mathrm{cia}^{24}$. Muula et al. salientam que ingerir bebida alcoólica nos últimos 12 meses se apresenta associado à ideação suicida ${ }^{17}$. Essa relação é evidenciada por outros estudos que revelam que o abuso/dependência de álcool, assim como o transtorno de conduta, são fatores contribuintes para tal relação 25 .

Quanto a outros comportamentos de risco à saúde relacionados à ideação suicida, no presente estudo se observa a associação entre o desfecho e o adolescente carregar revólver, faca ou canivete e ter se envolvido em brigas com comportamentos agressivos no último ano. A literatura científica também relaciona a ideação suicida com comportamentos agressivos. Cerel et al. indicam que o envolvimento em brigas está associado à ideação suicida na adolescência ${ }^{26}$. Segundo Alikasifoglu et al., tais comportamentos agressivos, além de estarem associados à ideação suicida, podem estar associados ao uso de substâncias como o álcool ${ }^{27}$. Dessa forma, a externalização de alguns comportamentos de risco à saúde pode ocorrer de maneira conjunta e se inter-relacionar, sendo importantes sinalizadores de risco acrescido de suicídio.

Cabe salientar que a presente pesquisa contou com um questionário autoaplicado para assegurar o sigilo dos dados e encorajar os jovens a serem o mais fidedignos com os dados relatados. Apesar disso, algumas limitações podem ser apontadas nesta investigação. O autorrelato não assegura o entendimento adequado das questões propostas. Ademais, a forma de aferição da ideação suicida pode ser considerada superficial para um desfecho de tal relevância, contudo é adequada para estudos epidemiológicos com grandes populações. A forma de seleção da amostra, o tamanho dela e a congruência de grande parte dos resultados encontrados com a literatura científica contribuem para salientar a qualidade e a credibilidade das informações utilizadas.

Com base nos resultados, sugerimos, como método de prevenção, trabalhos informativos nas escolas, direcionados a professores para que eles possam identificar pensamentos suicidas e comportamentos autodestrutivos. Além disso, viabilizar orientações aos responsáveis e encaminhamentos aos locais de assistência à saúde mental. Da mesma forma, evidencia-se a necessidade de campanhas educativas de grande divulgação, informando sobre a ideação suicida e as características usualmente relacionadas a tais contextos. Tais informações podem modificar os ambientes culturais e sociais onde o jovem está inserido, no sentido de tornar cuidadores e responsáveis agentes promotores de saúde. Assim, os fatores associados à ideação suicida observados na presente investigação podem contribuir para a caracterização 
dos contextos em que surge a ideação suicida juntamente com as diretrizes e ações preventivas indicadas na Política Nacional de Redução da Morbimortalidade por Acidentes e Violências ${ }^{10}$ e no Manual de Prevenção ao Suicídio dirigido aos profissionais das equipes de saúde mental'".

Portanto, os resultados apresentados indicam a necessidade de futuras pesquisas e sugerem a realização de investigações longitudinais com intervenções preventivas, a fim de acompanhar o desenvolvimento da ideação suicida nos adolescentes, observando a relação temporal e causal entre exposição e desfecho.

\section{CONCLUSÃO}

As estratégias de prevenção ao comportamento suicida devem focar a ideação suicida em jovens. Ademais, os resultados aqui expostos embasam a criação de projetos e programas que proporcionem discussões e ações sobre a influência de ideação suicida e demais fatores que possam prejudicar a saúde mental dos adolescentes. Tais programas preventivos devem preferencialmente atingir adolescentes de baixa escolaridade que apresentem comportamento agressivo e relatem uso de substâncias.

\section{AGRADECIMENTOS}

Agradecemos à Fundação de Amparo à Pesquisa do Estado do Rio Grande do Sul (FAPERGS) pelo apoio financeiro, bem como à Universidade Católica de Pelotas (UCPel) por oferecer bolsas de iniciação científica aos alunos que contribuíram para a execução da presente investigação.

\section{REFERÊNCIAS}

1. Brezo J, Paris J, Barker ED, Tremblay R, Vitaro F, Zoccolillo M, et al. Natural history of suicidal behaviors in a population-based sample of young adults. Psychol Med. 2007;37(11):1563-74.

2. Muza G, Bettiol H, Muccillo G, Barbieri M. Consumo de substâncias psicoativas por adolescentes escolares de Ribeirão Preto, SP (Brasil). I - Prevalência do consumo por sexo, idade e tipo de substância. Rev Saude Publica. 1997;31(1):21-9.

3. Mann JJ. A current perspective of suicide and attempted suicide. Ann Int Med. 2002;136:302-11.

4. Wichstrom L. Predictors of adolescent suicide attempts: a nationally representative longitudinal study of Norwegian adolescents. J Can Acad Child Adolesc Psychiatry. 2000;39:603-10.

5. Gould MS, Fisher P, Parides M, Flory M, Shaffer D. Psychosocial risk factors of child and adolescent completed suicide. Arch Gen Psychiatry. 1996;53:1155-62.
6. Chen PCY, Lee LK, Wong KC, Kaur J. Factors relating to adolescent suicidal behavior: a cross-sectional Malaysian school survey. J Adolesc Health. 2005;(37):337.

7. Baggio L, Palazzo LS, Aerts DRGC. Planejamento suicida entre adolescentes escolares: prevalência e fatores associados. Cad Saude Publica. 2009;25(1):142-50.

8. Silva VF, Oliveira HB, Botega NJ, Marín-León L, Barros MBA, Dalgalarrondo P. Fatores associados à ideação suicida na comunidade: um estudo de caso-controle. Cad Saude Publica. 2006;22(9):1835-43.

9. Reinherz HZ, Tanner JL, Berger SR, Beardslee WR, Fitzmaurice GM. Adolescent suicidal ideation as predictive of psychopathology, suicidal behavior, and compromised functioning at age 30. Am J Psychiatry. 2006;163:1226-32.

10. Portal.saude.gov [internet]. Brasília: Ministério da Saúde. Política Nacional de Redução da Morbimortalidade por Acidentes e Violências. 2001. Disponível em: <http://portal.saude. gov.br/portal/arquivos/pdf/portaria737.pdf>.

11. Bvsms.saude.gov [internet]. Brasília: Ministério da Saúde. Manual de Prevenção ao Suicídio dirigido aos profissionais das equipes de saúde mental. 2006. Disponível em: http:// bvsms.saude.gov.br/bvs/publicacoes/manual_editoracao.pdf.

12. Mari J, Williams P. A validity study of a psychiatric screeninng questionnaire (SRQ-20) in primary care in the city of São Paulo. Br J Psychiatry. 1986;148:23-6.

13. Januzzi PM, Baeninger R. Qualificação socioeconômica e demográfica das classes da escala Abipeme. Rev Administração. 1996;31(3):82-90.

14. Werlang BSG, Borges VR, Fensterseifer L. Fatores de risco ou proteção para a presença de ideação suicida na adolescência. R Interam Psicol. 2005;39(2):259-66.

15. Botega NJ, Marín-León L, Oliveira HB, Barros MBA, Silva VF, Dalgalarrondo P. Prevalências de ideação, plano e tentativa de suicídio: um inquérito de base populacional em Campinas, São Paulo, Brasil. Cad Saude Publica. 2009;25(12):2632-8.

16. Jatobá JDVN, Bastos 0 . Depression and anxiety in adolescents from public and private schools. J Bras Psiquiatr. 2007;56(3):171-9.

17. Muula AS, Kazembe LN, Rudatsikira E, Siziya S. Suicidal ideation and associated factors among in-school adolescents in Zambia. Tanzan Health Res Bull. 2007;9(3):202-6.

18. Rudatsikira E, Muula AS, Siziya S. Prevalence and associated factors of suicidal ideation among school-going adolescents in Guyana: results from a cross sectional study. Clin Pract Epidemiol Ment Health. 2007;23:3-13.

19. Souza LDM, Silva RA, Jansen K, Kuhn RP, Horta BL, Pinheiro RT. Suicidal ideation in adolescents aged 11 to 15 years: prevalence and associated factors. Rev Bras Psiq. 2010;32(1):3741.

20. An $H, A h n J H, B h a n g ~ S Y$. The association of psychosocial and familial factors with adolescent suicidal ideation: a population-based study. Psychiatry Res. 2010;177(3):318-22.

21. Kim MH, Jung-Choi K, Jun HJ, Kawachi I. Socioeconomic inequalities in suicidal ideation, parasuicides, and completed suicides in South Korea. Soc Sci Med. 2010;70:1254-61.

22. Taliaferro LA, Rienzo BA, Miller MD, Pigg RM Jr, Dodd VJ. High school youth and suicide risk: exploring protection afforded through physical activity and sport participation. J Sch Health. 2008;78(10):545-53.

23. Babiss $L A$, Gangwisch JE. Sports participation as a protective factor against depression and suicidal ideation in adolescents as mediated by self-esteem and social support.J Dev Behav Pediatr. 2009;30(5):376-84.

24. Juan W, Xiao-Juan D, Jia-Ji W, Xin-Wang W, Liang X. The associations between health risk behaviors and suicidal ideation and attempts in an urban Chinese sample of adolescents. J Affect Disord. 2010; doi:10.1016/j.jad.2010.02.121.

25. Borges G, Nock MK, Medina-Mora ME, Hwang I, Kessler RC. Psychiatric disorders, comorbidity, and suicidality in Mexico. J Affect Disord. 2010;124(1-2):98-107.

26. Cerel J, Roberts TA, Nilsen WJ. Peer suicidal behavior and adolescent risk behavior. J Nerv Ment Dis. 2005;193(4):237-43.

27. Alikasifoglu M, Erginoz E, Ercan 0, Uysal 0, Kaymak DA, Iter 0. Violent behaviour among Turkish high school students and correlates of physical fighting. Eur J Public Health. 2004;14(2):173-7. 